
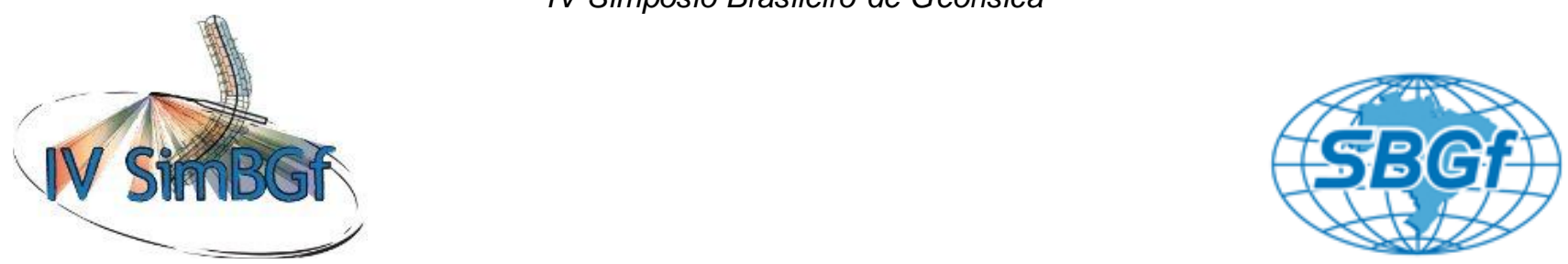

\title{
Dimensionamento de galeria de águas pluviais com os métodos de eletrorresistividade 2D e de GPR
}

Márcio Maciel Cavalcanti (marciom .cavalcanti@hotmail.com - UnB); Welitom Rodrigues Borges (welitom@unb.br - IG/UnB); Luciano Soares da Cunha(lucianosc@unb.br - IG/UnB); Eduardo Xavier Seimetz (edu.seimetz@gmail.com - Mestrando em Geociências Aplicadas - IG/UnB); Pedro Vencovski Nogueira(pvencovxky@gmail.com - Discente de Geologia - IG/UnB).

Copyright 2010, SBGf - Sociedade Brasileira de Geofísica

Este tex to foi preparado par a a apresentação no IV Simpósio Brasileiro de Geofísica, Brasília, 14 a 17 de novembro de 2010. Seu conteúdo foi revisado pelo Comite Técnico do IV SimBGf, mas não necessariamente representa a opinião da SBGf ou de seus associados. É proibida a reprodução total ou parcial deste material para propósitos comerciais sem pr évia autorização da SBGf.

\section{Resumo}

O crescimento das antigas cidades apresenta problemas em localizar galerias de águas pluviais. O uso dos métodos geofísicos, não evasivos, apresenta boa aplicabilidade em localizar e dimensionar estas tubulações. Utilizou-se o arranjo dipolo-dipolo e GPR com antenas blindadas de $200 \mathrm{MHz}$ e $400 \mathrm{MHz}$. Os resultados foram processados nos programas Res2dinv, versão 3.57 (eletrorresistividade) e REFLEXW® versão 5.5.1 (GPR). Os dados de eletrorresistividade mostram que os menores valores de resistividade ocorrem na área da tubulação de água pluvial. No processamento dos dados de GPR evidencia-se a eficiência na localização do topo e base da galeria.

\section{Introdução}

O presente estudo teve por objetivo a avaliação de métodos de eletrorresistividade 2D e Ground Penetrating Radar (GPR) na aplicabilidade de dimensionar estruturas enterradas para canalização de águas pluviais .

Nos processos de desenvolvimento das áreas urbanas antigas ocorre à dificuldade de determinar as redes de águas pluviais e de esgoto devido à perda/inexistência dos arquivos de cadastro. Conhecer as dimensões e a localização destas redes urbanas é de fundamental importância para a correta instalação de novas galerias que atendam as necessidades de ocupação urbana, devido ao aumento de áreas impermeabilizadas como o asfaltamento de ruas e avenidas. Esta problemática é rotina das companhias de água e esgoto na reestruturação dos canais de água pluvial tendo a busca das dimensões adequadas às galerias já existentes.

Para o levantamento deste estudo utilizou-se a galeria de águas pluviais, as margens do Lago Paranoá, dentro do Campus da Universidade de Brasília localizado no Distrito Federal. A área é destinada para prática de esportes náuticos e nas suas margens apresenta-se uma galeria de concreto armado, com $13 \mathrm{~cm}$ de espessura de parede lateral, $24 \mathrm{~cm}$ de espessura da parede superior e $300 \mathrm{~cm}$ de largura e altura (Figura 1). Desse modo a galeria de água pluvial, presente no Campus universitário de Brasília, confimará a aplicabilidade dos métodos elétricos (eletrorresistividade 2D) e do GPR na delimitação/identificação destas estruturas em subsuperfície.

\section{Metodologia}

A utilização de métodos geofísicos na identificação de estruturas geotécnicas subterrâneas já está consagrada em estudos de engenharia (Gallas, 2000).

Os métodos que apresentam a melhor eficácia na detecção de estruturas de concreto enterradas são os métodos da eletrorresistividade e o radar de penetração no solo.

O método da eletrorresistividade consiste basicamente em injetar correntes elétricas no solo e medir a diferença de potencial elétrico gerado pelo fluxo da corrente. De acordo com Dahlin e Zhou (2004), o arranjo dipolo-dipolo apresenta a melhor resolução no imageamento de estruturas pontuais presentes no subsolo.

Neste trabalho utilizou-se o equipamento multicanal Syscal Pro 72 (fabricado pela Iris Instruments), de propriedade do Laboratório de Geofísica Aplicada (LGAIG/UnB). Os eletrodos foram afixados no subsolo a cada 1 metro, obedecendo a distribuição de disposição de pontos do arranjo dipolo-dipolo. Foram usados 36 eletrodos de aço inox, e de acordo com a rotina de aquisição do equipamento (protocolo), foram investigados 37 níveis de profundidade, resultando na coleta de 756 pontos.

O GPR utiliza-se de ondas de rádio de alta freqüência (10 a $2500 \mathrm{MHz}$ ) para estudos de estruturas subsuperficiais (Annan, 1992). Sua antena transmissora emite um pulso para o subsolo, onde este pulso é refletido, refratado e difratado para uma antena receptora. Este sinal recebido é então processado por meio de digitalização sendo possível a visualização de padrões de reflexão relacionados a objetos enterrados no subsolo. Sua aplicação é bastante utilizada em estudos geológicos, arqueológicos, ambientais e geotécnicos.

$\mathrm{Na}$ aquisição dos dados de GPR foi utilizado o SIR3000 (fabricado pela Geophysical Survey Systems Inc.), de propriedade do Laboratório de Geofísica de Prospecção e Sensoriamento Remoto (LGPSR/UFC). O perfil de afastamento constante foi adquirido transversalmente a galeria de escoamento de água pluvial, com antenas blindadas de $200 \mathrm{MHz}$ e $400 \mathrm{MHz}$, 
espaçamento entre os traços de $2,5 \mathrm{~cm}$ e janela temporal de $150 \mathrm{~ns}$.
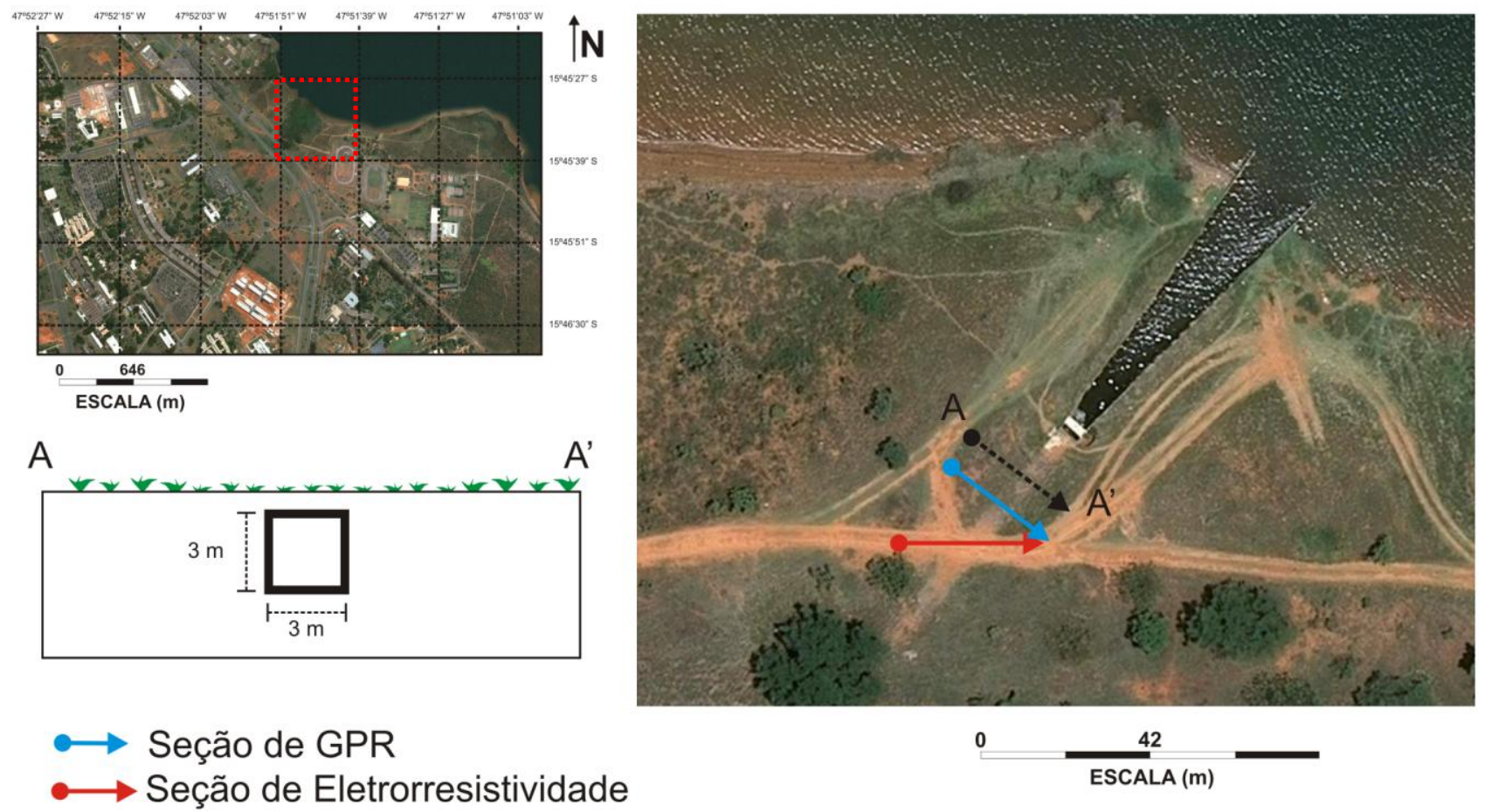

Figura 1 - Mapa de localização da área investigada com os métodos geofísicos e croqui esquemático da galeria de águas pluviais nas margens do Lago Paranoá, campus Darcy Ribeiro da UnB, Brasília/DF.

\section{Resultados}

A inversão dos dados de resistividade elétrica foi realizada no software Res2dinv, versão 3.57. A rotina de inversão utilizada foi a de contraste de suavização por mínimos quadrados (Sasaki, 1989), implementada no software. O método de otimização utilizado foi o Gauss Newton que recalcula as derivadas parciais da matriz jacobiana para todas as iterações. Este método aumenta o tempo de processamento, entretanto, apresenta melhores resultados quando há contrastes elevados de resistividade, ou seja, é melhor para detectar alvos pontuais com resistividades distintas (Loke e Dahlin, 2002).

A Figura 2 mostra os resultados da tomografia elétrica obtida sobre a área investigada. Os resultados da inversão mostram que houve um ajuste com um grande erro $(41,8 \%)$ entre a seção elétrica de campo (Figura 2a) e o dado sintético (Figura 2b). O modelo geoelétrico obtido (Figura 2c) mostra valores de resistividade elétrica entre $0,5 \mathrm{Ohm} . \mathrm{m}$ e $2500 \mathrm{Ohm} . \mathrm{m}$.

No modelo geoelétrico (Figura 2c) notam-se regiões com resistividade maior que $50 \mathrm{Ohm} . \mathrm{m}$ e uma área anômala com resistividade inferior. $\mathrm{Na}$ área condutiva nota-se uma anomalia circular (posição 16 metros do modelo).
A seção de GPR foi processada no Módulo 2D do software REFLEXW® versão 5.5.1 (Sandmeier, 2010) e todas as etapas do processamento foram desenvolvidas de forma a destacar a anomalia alvo deste trabalho.

Inicialmente, o dado de campo foi filtrado (dewow), eliminando-se os ruídos eletrônicos e estáticos inerentes ao sistema. O processamento envolveu: a deteminação da chegada da primeira onda (ajuste do tempo zero); aplicação de decaimento de energia; filtragens 1D (bandpass frequency), 2D (remoção do background) e conversão da escala de tempo para profundidade.

Para conversão dos perfis em profundidade foi realizado um ajuste hiperbólico de alguns pontos difratores encontrados ao longo do perfil. A velocidade obtida foi de $0,094 \mathrm{~m} / \mathrm{ns}$, utilizada na inversão dos dados (tempo para profundidade).

A Figura 3 é a seção de GPR após todas as etapas de processamento. Na mesma notam-se vários refletores subhorizontais de alta amplitude e alguns refletores hiperbólicos. Entre a posição de $10 \mathrm{~m}$ a $14 \mathrm{~m}$ ocorrem refletores horizontais de alta amplitude, de $0,5 \mathrm{~m}$ até a profundidade de 3,5 metros. 


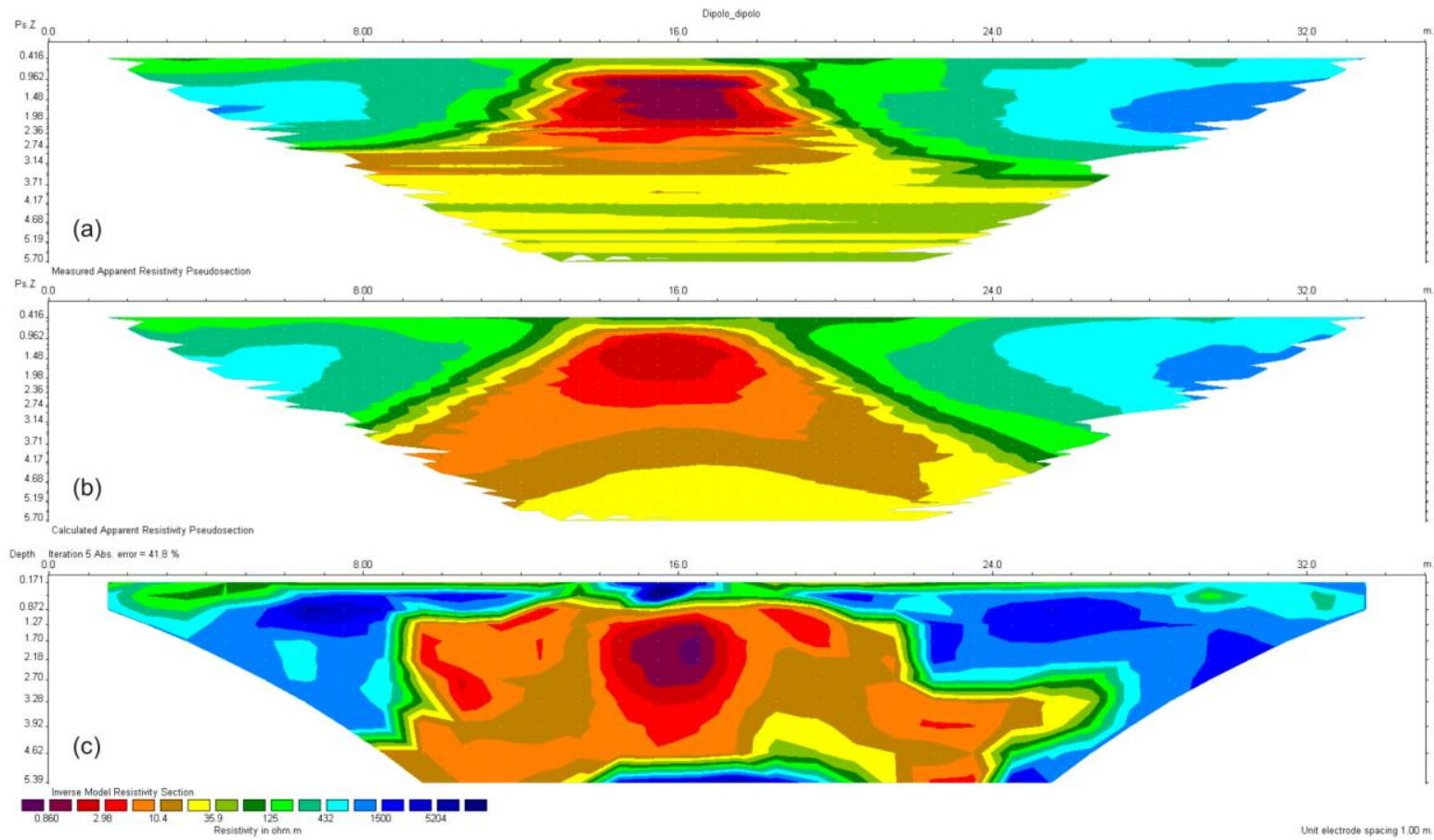

Figura 2 - Seções de eletrorresistividade sobre a área investigada. (a) Pseudo-seção de resistividade aparente dos dados de campo. (b) Dados sintéticos do ajuste do modelo. (c) Modelo de resistividade elétrica obtido após a inversão.

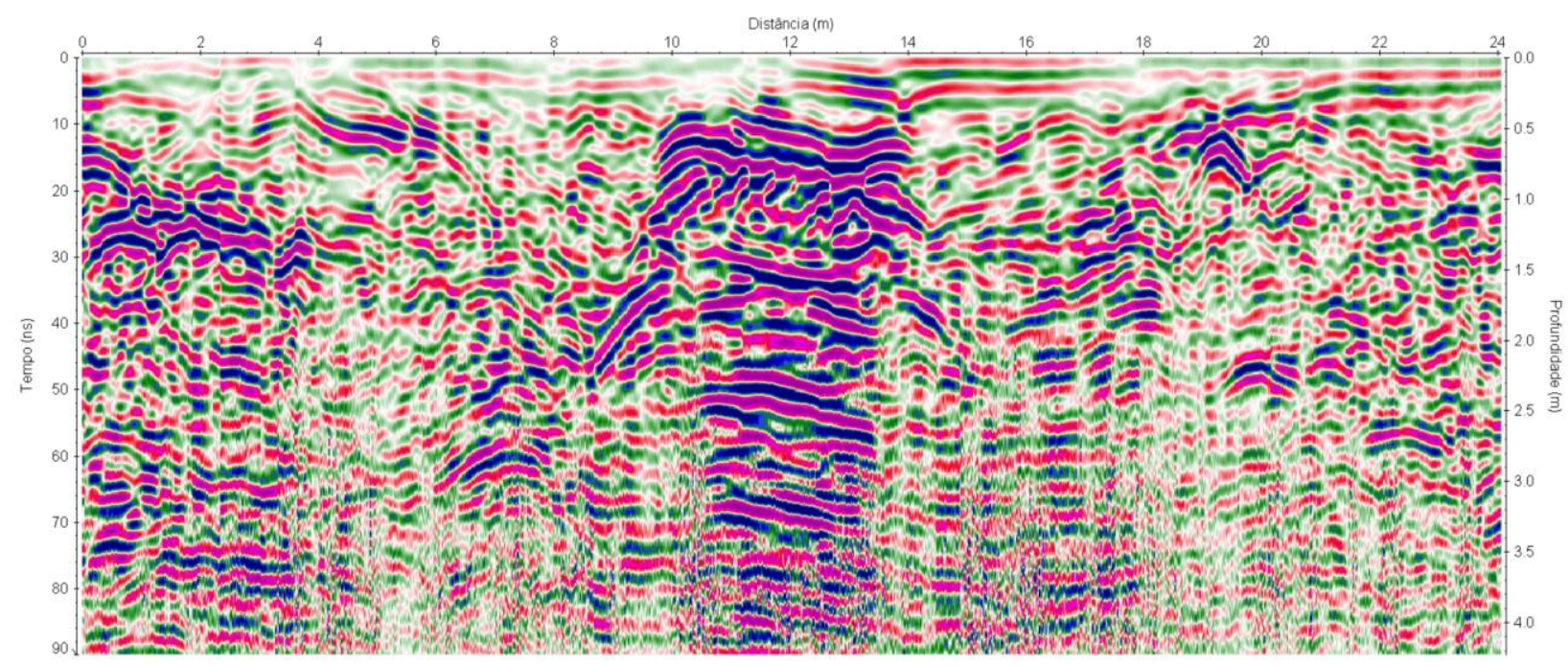

Figura 3 - Seção GPR obtida com a antena de $200 \mathrm{MHz}$ na área investigada.

\section{Discussão e Conclusões}

Os resultados de eletrorresistividade mostram que os menores valores de resistividade elétrica $(0.5$ a 10 Ohm.m), obtidos em campo, ocorrem na área da tubulação de água pluvial. Este efeito é provocado pela armação de ferro presente no interior do concreto, fazendo com que o fluxo de corrente elétrica fosse maior do que nas demais áreas (Figura 4).
O resultado de GPR (Figura 4) mostra eficiência na localização da galeria pluvial, possibilitando a identificação do topo (T) e base da mesma (B). Notam -se também reflexões múltiplas (Figura 4) provenientes da reflexão da onda eletromagnética no topo da galeria (M1), bem como da reflexão secundária da base da galeria (RM1).

Apesar da localização e dimensionamento não muito precisos da galeria pluvial, nota-se uma boa coerência nas interpretações. Todavia, ressalta-se que estes 
resultados só podem ser aplicados a estruturas geotécnicas presentes em baixas profundidades, pois 0 volume do objeto influenciará na resposta geofísica.

\section{Agradecimentos}

Ao doutorando Luis Anselmo do Curso de Engenharia Elétrica e ao técnico Péricles de Brito Macedo pelo auxílio na aquisição de dados em campo. Ao Laboratório de Geofísica Aplicada (LGAUnB) pela disponibilização dos equipamentos geofísicos.

\section{Referências}

ANNAN, A.P, 1992. Ground Penetrating Radar, Workshop Notes. Sensores e Software, Inc. Internal Report. pp.130.

DAHLIN, Torleif \& Bing, ZHOU. 2004. A numerical comparison of $2 \mathrm{D}$ resistivity imaging with ten electrode arrays. Geophysical Prospecting. № 52. pp.379-398.
GALLAS, José Domingos Franco, 2000. Principais métodos geoelétricos e suas aplicações em prospecção mineral, hidrogeologia, geologia de engenharia e geologia ambienta. Tese de Doutorado, Universidade Estadual Paulista. Rio Claro, SP.

LOKE M.H.; DAHLIN, T. 2002. A comparison of the Gauss-Newton and quasi-Newton methods in resistivity imaging inversion, Joumal of Applied Geophysics, Vol. 49(3): 149-162.

SANDMEIER, K.J., 2006. REFLEXW Version 4.2, Windows $9 \times / 2000 / N T$. Program for the processing of seismic, acoustic or electromagnetic reflection, refraction and transmission data. Manual do Software, Karlsruhe, Germany, 209p.

SASAKI, Y. 1989. Two-dimensionas joint inversion of magnetotelluric and dipole-dipole resistivity data. Geophysics, Vol. 54(2):254-254.

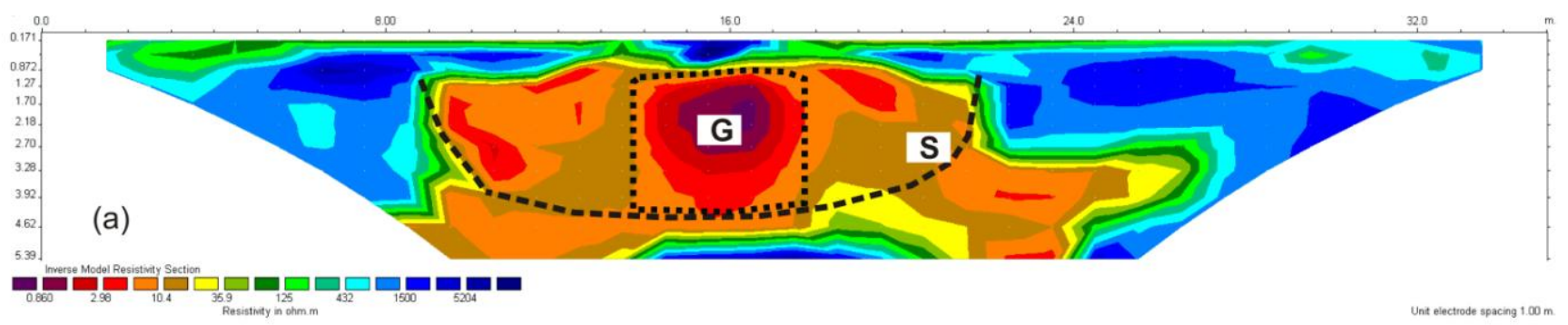

(b)

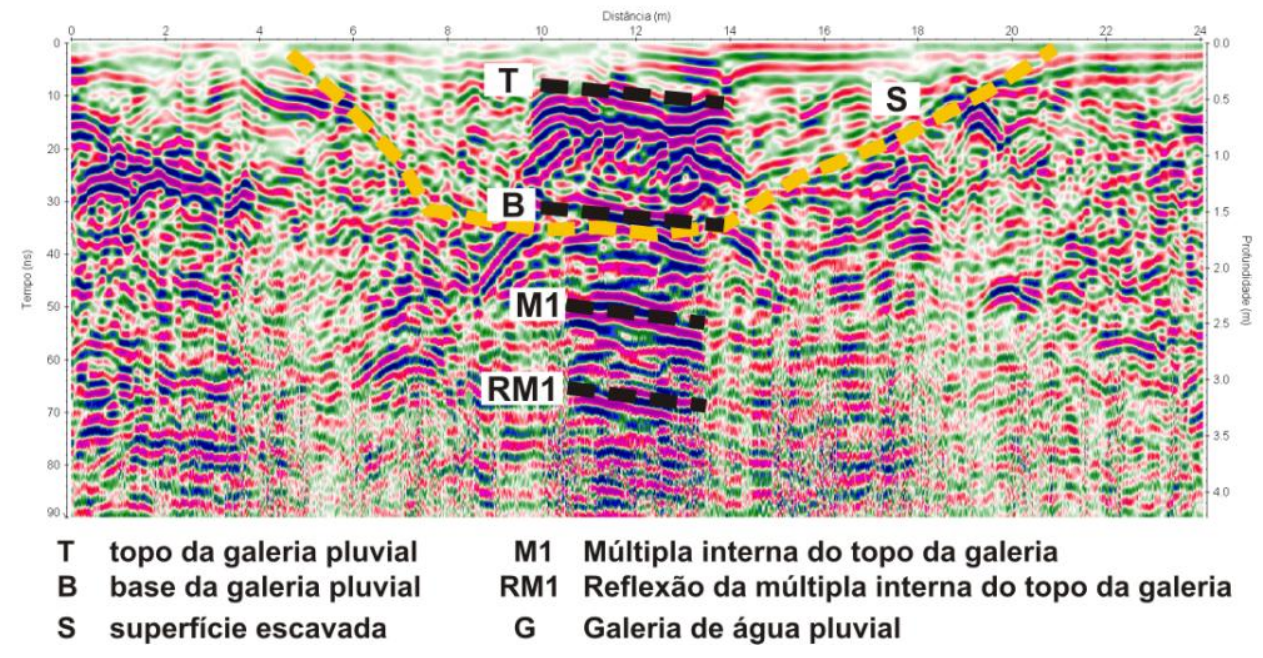

Figura 4 - Seções geofísicas com as interpretações sobrepostas. (a) Seção elétrica evidenciando as anomalias relacionadas a galeria de águas pluviais - G, e a superfície de escavação - S. (b) Seção GPR evidenciando o topo (T) e base (B) da galeria de águas pluviais, bem como das reflexões múltiplas, e a superfície de escavação (S). 